\title{
Prevalência de pessoas com deficiência física e acesso ao serviço de reabilitação no Brasil
}

\author{
Prevalence of people with disabilities and acess to \\ rehabilitation service in Brazil
}

Emanuele Farencena Franchi, ${ }^{1}$ Viviane Dutra Piber, ${ }^{1}$ Clarissa Maciel Selau, ${ }^{1}$ Michelle Hillig Schmidt, ${ }^{1}$ Priscila da Silva Soares, ' Louise Bertoldo Quatrin'

${ }^{1}$ Centro Universitário Franciscano, Santa Maria, RS, Brasil.

Recebido em: 24/02/2017 / Aceito em: 24/05/2017 / Publicado em: 30/06/2017

emanuele.franchi@hotmail.com

\section{RESUMO}

A Organização Mundial da Saúde (OMS) afirma que pelo menos $10 \%$ da população mundial apresenta algum tipo de deficiência, tendo estes direitos que, asseguram o acesso à reabilitação. Objetivo: conhecer a prevalência de pessoas com deficiência física que frequentam algum serviço de reabilitação no Brasil. Método: o estudo transversal e ecológico, compara dados do Instituto Brasileiro de Geografia e Estatística (IBGE) referentes à Pesquisa Nacional de Saúde (PNS), do ano de 2013. Resultados: apenas $18,4 \%$ do total de pessoas com deficiência física frequentam algum serviço de reabilitação, $20,2 \%$ das que residem na zona urbana, e $8,9 \%$ na zona rural, sendo a maior frequência de crianças com idade entre 0 e 9 anos e menor de pessoas com idade entre 40 e 59 anos. Na região Sul, $23 \%$ frequentam algum serviço, e na região Nordeste somente $15,9 \%$. O acesso destas pessoas aos serviços pode estar relacionado à ausência ou insuficiência de acessibilidade ainda presente em muitos serviços de saúde e sua distribuição, assim como a falta de orientação por parte dos profissionais. Conclusão: o número de pessoas com deficiência física que frequentam serviços de reabilitação ainda é baixo, mesmo diante das políticas e do Sistema Único de Saúde (SUS), que garantem o acesso a esses serviços de forma gratuita.

Palavras-chave: Pessoas com Deficiência; Serviços de reabilitação; Políticas públicas de saúde; Acesso aos Serviços de Saúde.

\section{ABSTRACT}

The World Health Organization (WHO) states that at least $10 \%$ of the world population has some kind of disability; these people has rights that ensure access to rehabilitation. Objective: to determine the prevalence of people with disabilities who attend rehabilitation services in Brazil. Method: cross-sectional and ecological study comparing data from the Geography and Statistics Brazilian Institute (IBGE) corresponding to National Health Survey (PNS) from 2013. Results: only $18.4 \%$ of physically disabled people attend some rehabilitation services $120.2 \%$ of these are living in urban areas, and $8.9 \%$, in rural areas). The higher prevalence is of children aged 0 to 9 years, and lowest of people aged between 40 and 59 years. In the South region $23 \%$ attend a service and in the Northeast, only $15.9 \%$. These people's access to services might be related to the absence or insufficiency accessibility still present in many health services, and their distribution as well as lack of professional guidance. Conclusion: the number of people with physical disabilities who attend rehabilitation services is still low, even in the face of policies and the Unified Health System (SUS) that ensure access to these services free of charge.

Keywords: People with Disabilities; Rehabilitation Services; Public health policies; Access to Health Services. 


\section{INTRODUÇÃO}

A Organização Mundial da Saúde (OMS) afirma que, pelo menos $10 \%$ da população mundial, apresenta algum tipo de deficiência. ${ }^{1}$ Sabe-se da dificuldade de definição do conceito de deficiência, pois é complexo, dinâmico e questionável, entretanto habituou-se a denominar impairments, disability, handicap, disfuncion, discapacidade, deficiência como a diferença entre indivíduos que possuem lesão, função prejudicada ou dificuldade no desempenho de atividades. Esta denominação gerou muitos debates históricos e linguísticos como deficiência, incapacidade, invalidez. Esses termos fazem parte de um longo processo de ressignificação que demonstram a dificuldade de conceituação entre deficiência e normalidade. ${ }^{2}$

Segundo a Classificação Internacional de Funcionalidade, Incapacidade e Saúde (CIF) da Organização Mundial da Saúde, "as deficiências são problemas nas funções ou estruturas do corpo, tais como um desvio importante ou uma perda." ${ }^{3}$ No entanto, o aspecto mais importante a ser levado em consideração é a funcionalidade dos indivíduos, pois além das questões orgânicas e corporais, a pessoa é considerada um ser social. ${ }^{4}$ Desta forma, as diferenças corporais, sendo ou não classificadas como deficiências, são expressões da diversidade humana. ${ }^{4}$ A existência de corpos díspares sempre despertou curiosidade, espanto, indiferença, exclusão, na maioria das vezes, deixando as pessoas diferentes à margem, impossibilitando em muitos momentos sua participação social e o cumprimento de seus direitos de cidadãos. ${ }^{4}$

No Brasil, as pessoas com deficiência possuem seus direitos assegurados na Constituição da República Federativa do Brasil de 1988, assim como todos os cidadãos brasileiros. Dentre estes, destaca-se o direito de acesso à saúde. Dois princípios doutrinários do Sistema Único de Saúde demonstram esse direito, a universalidade que é a garantia de atenção por parte do sistema, a todo e qualquer cidadão; neste contexto todo cidadão passa a ter direito de acesso a todos os serviços públicos de saúde, e a integralidade garante o atendimento de forma integral nos três níveis de assistência. ${ }^{5}$

Em 2008, a Organização Mundial da Saúde cria a Política Nacional de Saúde da Pessoa com deficiência, estabelecendo responsabilidades institucionais, criando condições para reabilitação das pessoas com deficiência e diretrizes para o cuidado, assistência, proteção e garantia de saúde de forma integral. ${ }^{6}$ Entende-se por reabilitação, o conjunto de ações e serviços que visam contribuir para a inclusão, restabelecer a capacidade funcional e o desempenho humano, após a instalação de dano ou enfermidade. ${ }^{7}$ A reabilitação e o acesso a esses serviços de saúde tratam-se de reconhecer que historicamente é um espaço de interconexão de elementos relacionados à saúde, limites e possibilidades. ${ }^{2}$

Através da portaria $n^{\circ} 793$ de 24 de abril, de 2012, institui-se uma Rede de Cuidados à Pessoa com Deficiência, contendo diretrizes que ampliam o acesso, articulação e criação de novos pontos de atenção à saúde. ${ }^{8}$ Dessa forma, percebe-se que a sociedade, a gestão, e as políticas públicas têm se preocupado em garantir o acesso integral e com qualidade a esta população, respeitando o direito das pessoas com deficiência e preocupando-se com sua saúde.

Diante disso, esta pesquisa teve como objetivo co- nhecer a prevalência de pessoas com deficiência física nas diferentes regiões do Brasil, de acordo com a situação de domicílio e grupos etários e a prevalência de pessoas com deficiência física que frequentam algum serviço de reabilitação de acordo com os mesmos critérios.

\section{MÉTODO}

O estudo do tipo transversal e ecológico, comparou dados referentes à população com deficiência física e o acesso destas aos serviços de reabilitação, de acordo com a situação de domicílio, grupos etários e regiões do Brasil. ${ }^{9}$

Os dados populacionais e de ocorrência de deficiência física foram extraídos do site do Instituto Brasileiro de Geografia e Estatística (IBGE), referentes à Pesquisa Nacional de Saúde (PNS) de 2013, com divulgação dos dados em 2015. Trata-se de uma pesquisa domiciliar, integrante do Sistema Integrado de Pesquisas Domiciliares - SIPD, contudo com desenho próprio, elaborado para coletar informações de saúde da população..$^{10} \mathrm{De}$ acordo com a pesquisa supracitada, foram classificadas pessoas com deficiência física, aquelas com alteração completa ou parcial de um ou mais segmentos do corpo humano, acarretando o comprometimento das funções física, neurológica e/ou sensorial. A pesquisa também investigou se as pessoas com deficiência frequentavam algum serviço de reabilitação em saúde, composto por equipes multiprofissionais de assistência interdisciplinar. Este caracteriza-se como serviço de reabilitação clínico-funcional das pessoas com deficiência, que contribui para a melhoria de suas condições de vida, sua integração social, ampliação das potencialidades laborais e independência nas atividades de vida diária. ${ }^{10}$

Durante a realização da pesquisa, esta classificação se deu através das seguintes perguntas ao entrevistado: “Qual deficiência física?", classificando-se dentre as opções supracitadas e "Frequenta algum serviço de reabilitação devido à deficiência física?", optando-se por sim ou não..11

Inicialmente, foi realizado o levantamento e tabulação dos dados referentes à população com deficiência física no Brasil, no ano de 2013. Juntaram-se a esses números, outros referentes às pessoas com deficiência física, que frequentam algum serviço de reabilitação, em relação a este mesmo período. Por se tratar de dados secundários, a pesquisa dispensou aprovação do Comitê de Ética em Pesquisa. Estes dados foram classificados de acordo com a situação de domicílio, grupos etários e regiões do Brasil. A seguir, foram desenvolvidos gráficos referentes a estes valores e agregados ao arquivo.

\section{RESULTADOS}

A Figura 1 mostra os dados da prevalência de pessoas com com deficiência física (DF) no Brasil e as pessoas com DF que frequentam algum serviço de reabilitação de acordo com a situação de domicílio. Verificou-se que existem 2.651 milhões de pessoas com DF no Brasil, sendo que destas 2.246 milhões residem na zona urbana e 405 mil na zona rural. O total de pessoas com DF, que frequentam serviços de reabilitação no Brasil, é 489 mil, destas 453 mil são da zona urbana e apenas 36 mil da zona rural. Observa-se que apenas $18,4 \%$ do total de pessoas com DF frequentam algum serviço de reabilitação; $20,2 \%$ das 


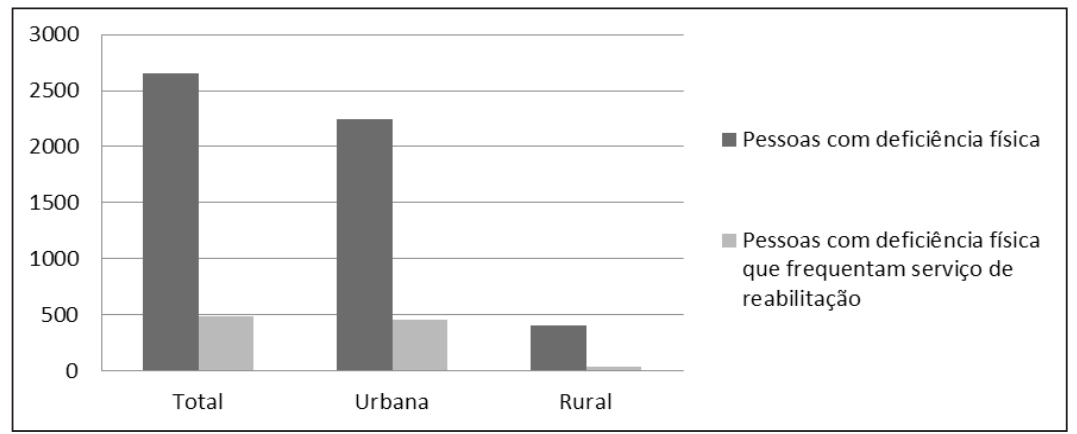

Figura 1 - Total de pessoas com Deficiência Física (DF) e pessoas com DF que frequentam serviços de reabilitação no Brasil.

que residem na zona urbana frequentam, e apenas $8,9 \%$ da zona rural utilizam esses serviços.

A figura 2 mostra a comparação de dados entre pessoas com DF e estas que frequentam serviços de reabilitação de acordo com os grupos etários e situação de domicílio. O número de crianças com DF de 0 a 9 anos que residem na zona urbana é de 101 mil; destas 58 mil $(57,6 \%)$ frequentam algum serviço de reabilitação, enquanto na zona rural o número de crianças com DF é de 22 mil; destas 6 mil $(27,1 \%)$ frequentam serviço de reabilitação. Na faixa etária de 40 a 59 anos, observa-se na zona urbana 787 mil pessoas com DF, sendo que $128 \mathrm{mil}(16,2 \%)$ frequentam serviços de reabilitação; na zona rural, o número de pessoas com DF é de 167 mil e 18 mil (10,5\%) frequentam o mesmo tipo de serviço. Observa-se a disparidade entre o número de crianças com DF, que frequentam os serviços de reabilitação, em relação à faixa etária de 40 a 59 anos, em que se observa maior frequência de crianças em serviços de reabilitação. Nesta figura, observa-se semelhança de dados entre as faixas etárias de 40 a 59 anos e 60 anos ou mais.

Na figura 3, observam-se o número de pessoas com DF e o número destas que frequentam serviço de reabilitação nas diferentes regiões do Brasil. Verifica-se que na região Nordeste apenas $15,9 \%$ das pessoas com DF frequentam algum serviço de reabilitação, diferentemente da região Sul, onde $23 \%$ frequentam estes serviços, observando-se maior assiduidade na região Sul do Brasil.

\section{DISCUSSÃO}

As políticas de saúde no Brasil foram se desenvolvendo a partir de movimentos que buscavam um modelo de saúde mais democrático e universal. A integralidade faz parte desse movimento por desacreditar na medici-

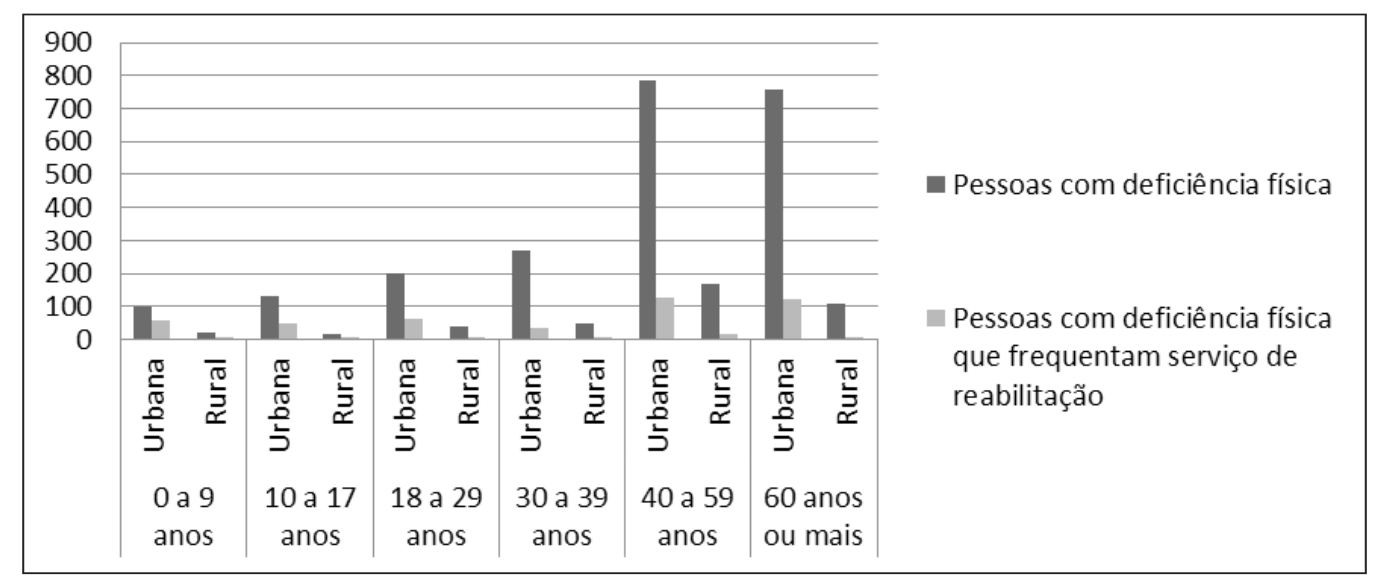

Figura 2 - Total de pessoas com Deficiência Física (DF) e pessoas com DF que frequentam serviços de reabilitação no Brasil de acordo com grupos etários e situação de domicílio.

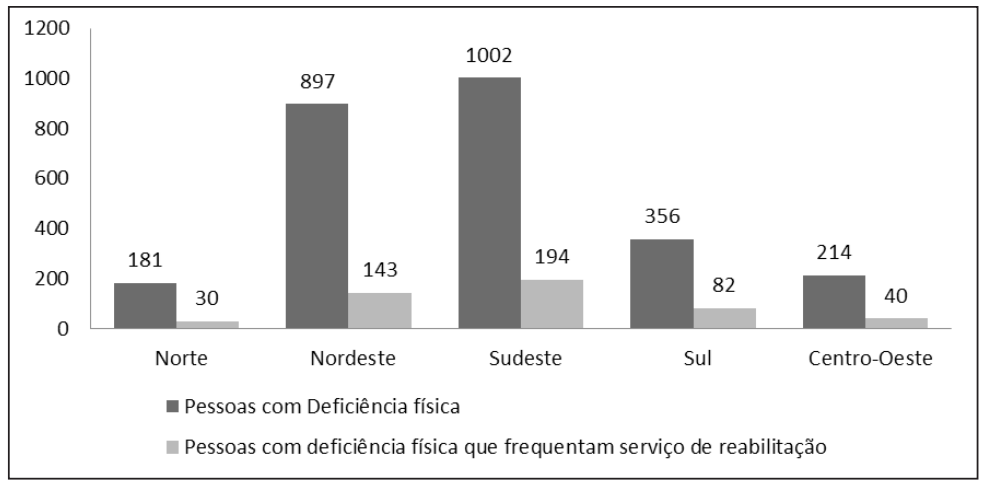

Figura 3 - Pessoas com Deficiência Física (DF) e pessoas com DF que frequentam serviços de reabilitação de acordo com as regiões do Brasil. 
na fragmentária e acreditar na diretriz do atendimento integral, onde se deve ter a articulação e continuação de ações e serviços de assistência à saúde. ${ }^{12}$

Baseado nos princípios da integralidade, universalidade e equidade, o SUS, com objetivo de promover melhor qualidade de vida e autonomia às pessoas com deficiência, oferece garantia de atenção à saúde, reabilitação, acesso a órteses, próteses e meios auxiliares de locomoção. ${ }^{13}$ Estes direitos estão assegurados desde a Constituição Federal de 1988, em diversos instrumentos legais, tal como a Política Nacional da Pessoa com Deficiência que reitera o quanto importante é esse acesso aos serviços de saúde, estabelecendo algumas normas e orientando quanto a atenção à saúde e o acesso à reabilitação que esses indivíduos tem direito, ${ }^{14}$ assim como na Portaria $\mathrm{n}^{\circ} 793$ de 24 de abril de 2012, que tem como diretriz a garantia de acesso e qualidade dos serviços, ofertando cuidado integral e assistência multiprofissional e interdisciplinar às pessoas com deficiência. ${ }^{8}$ Essas políticas tratam de colocar em pauta os conceitos propostos pelo SUS, na ideia de atenção à saúde não dicotomizada e sim de um acesso universal e igualitário à saúde de todos os cidadãos.

No entanto, as políticas de saúde brasileiras fomentam que toda a pessoa com deficiência tem direito ao acesso a serviços de reabilitação, visando melhorar a funcionalidade, e promover maior autonomia e independência. ${ }^{15}$ Durante a reabilitação, a intencionalidade, tanto pode ser restrita à restauração de funções, quanto pode vincular-se ao processo de participação social da pessoa com deficiência. ${ }^{2}$ Diante da existência de tantas políticas e de que a maioria saiba da importância de frequentar serviços de saúde, da população com DF existente no Brasil, apenas 18,5\% frequentam serviços de reabilitação. Observa-se o baixo acesso destas pessoas aos serviços, podendo ocasionar o agravamento de suas condições físicas, refletindo diretamente na autonomia e independência.

Em alguns casos, estes números reduzidos podem se dar devido à procura por atendimento de reabilitação que não ocorre diante da falta de orientações e encaminhamentos no momento da alta hospitalar, deixando as pessoas inseguras e desassistidas ao retornarem ao domicílio. Algumas frequentam serviços de reabilitação vinculados a convênios particulares/médicos, o que muitas vezes é desarticulado do contexto sociocultural do indivíduo, visando somente a melhoria da condição física do mesmo, que quando recebe alta do serviço ou é desligado, retorna para o domicílio sem perspectivas de inserção em atividades comunitárias, muitas vezes vivendo em situação de isolamento domiciliar. Diante disso, os profissionais de saúde envolvidos nesse contexto, devem dar maior atenção à implementação de estratégias de intervenção que promovam a assistência dessas pessoas também no âmbito territorial e comunitário. ${ }^{16}$

Outra dificuldade de acesso pode estar relacionada à ausência ou insuficiência de acessibilidade ainda presente em muitos serviços de saúde. A maioria das Unidades Básicas de Saúde (UBS) nas regiões Sul e Nordeste do Brasil não atendem às normas de acessibilidade da Associação Brasileira de Normas Técnicas (ABNT) para a população idosa e pessoas com deficiência, sendo preocupante a alta prevalência de barreiras arquitetônicas, pois estas garantem condições de mo- bilidade para que as pessoas com deficiência tenham acesso a estes serviços com autonomia e segurança. ${ }^{17}$ Siqueira et al., verificaram que $59,8 \%$ dos prédios não eram adequados para o acesso de idosos e pessoas com deficiência física. Em relação à inadequação de prédios, degraus, rampas, calçadas, corrimãos nas unidades e banheiros adaptados não observaram diferenças significativas entre a região Sul e Nordeste do Brasil, porém na região Nordeste $82,9 \%$ das unidades não disponibilizava de cadeira de rodas para aqueles que necessitassem. ${ }^{17}$ No presente estudo, o número de pessoas com DF residentes na região Sul que frequentam algum serviço de reabilitação ainda é baixo em relação ao total da população, sendo que dentre todas as regiões do Brasil, é a que apresenta maior adesão aos serviços de reabilitação. Já, na região Nordeste, a mais baixa em relação as outras regiões, este número é ainda menor, podendo este dado estar associado às questões de acessibilidade registradas nestas regiões.

Segundo a Organização Mundial da Saúde, as pessoas com deficiência possuem o direito de utilizar todo e qualquer serviço de assistência a saúde, ${ }^{2}$ e não somente em instituições específicas de reabilitação, deve ser assegurado o atendimento na rede de serviços, contemplando os diversos níveis de complexidade e especificidades. ${ }^{13}$ Para tanto, os serviços de atenção básica, atenção especializada em reabilitação e atenção hospitalar e de urgência e emergência, são articulados entre si, garantindo a integralidade do cuidado e o acesso regulado, observadas as especificidades para garantia da equidade a estes usuários. Os serviços de atenção especializada em reabilitação têm como um de seus objetivos, manutenção da função, além de recuperação da função perdida, assim como realizar a inclusão sociocultural dessas pessoas e autonomia na vida diária das mesmas. ${ }^{18}$

Além disso, deve-se atentar à distribuição destes serviços, pois ocorre centralidade de serviços na cidade, onde a frequência periódica condiciona-se à inviabilidade de deslocamento e a qualidade de resolutividade dos serviços. ${ }^{19}$ Percebe-se diante deste estudo, que 20,2\% das pessoas com DF, que residem na zona urbana, frequentam algum serviço, e apenas $8,9 \%$ das residentes na zona rural; estes encontram maior dificuldade de acesso, tanto pelo enfoque geográfico, como funcional e econômico. No entanto, segundo a Lei $n^{\circ} 13.146$, de 6 de julho de 2015, que institui a Lei Brasileira de Inclusão da Pessoa com Deficiência, é um dos direitos da pessoa com deficiência durante o processo de reabilitação, a prestação de serviços próximo ao seu domicílio, inclusive na zona rural, respeitadas a organização das redes de atenção à saúde nos territórios locais e as normas do sistema único de saúde (SUS). ${ }^{20}$

No estudo também foi observado que o número de crianças que frequentam serviços de reabilitação é consideravelmente maior do que os adultos na faixa de 40 a 59 anos. Apesar das políticas para pessoas com deficiência, podemos observar que há um maior investimento pela parte dos pais, pois a atuação entre serviços de reabilitação e os familiares trazem inúmeros benefícios para o processo de recuperação e estimulação. ${ }^{21}$ Sabe-se da dificuldade de compreensão de alguns pais acerca da deficiência do filho e isso pode levar a um processo de desorientação sobre qual serviço buscar, porém os profissionais envolvidos nesse processo deve- 
riam orientar os pais sobre os prejuízos para a criança.

O reduzido número de adultos que frequentam serviços de reabilitação pode estar relacionado à falta de acesso, orientação sobre a importância e frustrações anteriormente vividas nestes atendimentos. O papel dos familiares não é apenas de suporte, mas sim de facilitadores, respeitando suas facilidades e limitações durante a reabilitação, estimulando a pessoa com DF a conquistar o máximo de independência. ${ }^{22}$

Diante do exposto, é de suma importância a orientação dos familiares e/ou cuidadores, para que estes cidadãos consigam participar mais efetivamente da sociedade e manter sua qualidade de vida. Faz-se necessário uma reestruturação nos modelos de intervenção que venham atender à demanda da população que está segregada da sociedade, que as políticas de saúde estejam em discussão e na busca por mais ações de promoção e prevenção de saúde, redes de serviços de atenção primária e especializada, que promovam assistência em reabilitação.

Este estudo apresenta como limitação o desenho da pesquisa, visto que esta analisou dados secundários; devido a isso a qualidade da coleta dos dados não pode ser garantida.

\section{CONSIDERAC̣ÕES FINAIS}

Pode-se observar a baixa adesão das pessoas com DF aos serviços de reabilitação, mesmo diante das políticas e do Sistema Único de Saúde (SUS) que garantem o acesso a esses serviços de forma gratuita, devendo-se investir em maior promoção e divulgação de ações que incentivem o acesso a todos os níveis de atenção à saúde.

Observa-se também a importância da articulação entre os profissionais que integram os serviços de reabilitação para que juntos consigam manter maior contato com outros setores, facilitando o acesso das pessoas com DF aos serviços, ampliando a autonomia e independência desta população, assim como a inclusão sociocultural dos mesmos.

\section{REFERÊNCIAS}

1. Organização Mundial de Saúde. Relatório Mundial sobre a deficiência- World Reporton Disability. Secretaria de Estado dos direitos da pessoa com deficiência. São Paulo, 2011.

2. Pereira SO. Reabilitação de pessoas com deficiência física no SUS: elementos para um debate sobre integralidade. [dissertação]. Salvador (BA):Universidade Federal da Bahia; 2009.

3. Organização Mundial de Saúde. CIF: Classificação Internacional da Funcionalidade. Direção geral de saúde. Lisboa, 2004.

4. Tonús D, Wagner LC. Inclusão em escolas regulares: percepção de pais e professores de crianças e adolescentes com deficiência. Ciência em Movimento 2013;15(31):33-45.

5. Brasil. Constituição (1988). Constituição da República Federativa do Brasil: promulgada em 5 de outubro de 1988: atualizada até a Emenda Constitucional n. 20, de 15-121998. 21. ed. São Paulo: Saraiva, 1999.

6. Brasil, Ministério da Saúde. Secretaria de Atenção a Saúde.
Política Nacional da Pessoa Portadora de Deficiência. Editora do Ministério da Saúde. Brasília 2008.

7. Brasil. Ministério da Saúde. Manual de Legislação de Saúde da Pessoa com Deficiência. Secretaria de Atenção à Saúde. Brasília (DF), 2006.

8. Brasil. Ministério da Saúde. Portaria $n^{\circ} 793$, de 24 de abril de 2012. Institui a Rede de Cuidados à Pessoa com Deficiência no âmbito do Sistema Único de Saúde. Diário Oficial da União, Brasília (DF), 2012.

9. Carvalho ER, Rocha HAL. Estudos epidemiológicos. Faculdade de Medicina da Universidade Federal do Ceará. 2010. Disponível: http://www.epidemio.ufc.br/files/05estudosepidemiologicos. pdf. Acessado em 17 dez 2016

10. Instituto Brasileiro de Geografia e Estatística (IBGE). Pesquisa Nacional de Saúde: ciclos de vida: Brasil e grandes regiões. Ministério da Saúde Rio de Janeiro, 2015.

11. Instituto Brasileiro de Geografia e Estatística(IBGE). Manual: Pesquisa Nacional de Saúde, 2013.

12. Paim JS. Desafios para a saúde coletiva no século $X X I$. Salvador (BA): EDUFBA, 2006. Disponível:https://repositorio. ufba.br/ri/bitstream/ri/7078/1/Paim\%20J.\%20Desafio\%20 da\%20Saude\%20Coletiva.pdf.

13. Santos, JS. Pessoas com deficiências: uma breve análise sobre o centro de reabilitação de pessoas portadoras de deficiência (CERPPOD) - Patos/ PB [monografia]. Campina Grande (PB): Universidade Estadual da Paraíba; 2012.

14. Brasil, Ministério da Saúde. Secretaria de Atenção a Saúde. Política Nacional da Pessoa Portadora de Deficiência. Editora do Ministério da Saúde. Brasília (DF) 2008.

15. Brasil. Viver sem Limite - Plano Nacional dos Direitos da Pessoa com Deficiência. Secretaria de Direitos Humanos da Presidência da República (SDH/PR), Secretaria Nacional de Promoção dos Direitos da Pessoa com Deficiência (SNPD),4 ed. 2013.

16. Aoki M, Oliver FC, Nicolau SM. Considerações acerca das condições de vida das pessoas com deficiência a partir de um levantamento em uma unidade básica de saúde de um bairro periférico do município de São Paulo. O Mundo da Saúde. 2011;35(2):169-78.

17. Siqueira FCV, Facchini LA, Silveira DS, Piccini RX, Thumé E, Tomasi E. Barreiras arquitetônicas a idosos e portadores de deficiência física: um estudo epidemiológico da estrutura física das unidades básicas de saúde em sete estados do Brasil. Ciência \& Saúde Coletiva 2009;14(1):39-44. doi: 10.1590/S1413-81232009000100009

18. Brasil. Ministério da Saúde. Avanço das políticas públicas para as pessoas com deficiência: uma análise a partir das conferencias internacionais. 1 ed. Secretaria dos Direitos Humanos. Brasília (DF), 2012.

19. Alcântara LR, Lopes MJM. Estrutura de serviços e acesso a consumos em saúde por idosos em um contexto rural do Sul Brasil. REDES 2012;17(1):94-114. doi: 10.17058/redes.v17i1.1719

20. Brasil. Senado Federal. Estatuto da Pessoa com Deficiência. Lei Brasileira de Inclusão No 13.146, de 6 de julho de 2015. Diário Oficial da União. Brasília (DF), 2015.

21. Canho PGM, Neme CMB, Yamada M. O. A vivência do pai no processo de reabilitação da criança com deficiência auditiva. Estudos de Psicologia 2006;23(3):261-269. doi: 10.1590/S0103-166X2006000300005

22. Rocha SA, Avila MAG, Bocchi SCM. Influência do cuidador informal na reabilitação do idoso em pós-operatório de fratura de fêmur proximal. Rev Gaúcha Enferm 2016;37(1):1-9.

Como citar: FRANCHI, Emanuele Farencena et al. Prevalência de pessoas com deficiência física e acesso ao serviço de reabilitação no Brasil. Cinergis, Santa Cruz do Sul, v. 18, n. 3, maio 2017. ISSN 2177-4005. Disponível em: <https://online.unisc.br/seer/index.php/cinergis/article/view/8783>. Acesso em: 22 jun. 2017. doi:http://dx.doi.org/10.17058/cinergis.v18i3.8783. 